\title{
Optimal Design of Heatsink for 3 Phase Voltage Source Inverter System by Using Average Method
}

\author{
S.E. Cho \\ Department of Electrical Engineering, Seoil University Korea \\ Seoil University gil 22 Jungnang-Gu, Seoul, Korea \\ Tel: 82-2-490-7240 E-mail:secho@seoil.ac.kr \\ S.J. Park \\ Department of Electrical Engineering, Chonnam Nationnal University Korea \\ Yongbong-ro 77, Bug-gu, Kwangju, Korea \\ Tel: 82-62-530-0748_E-mail:sjpark1@chonnam.ac.kr
}

\begin{abstract}
In this paper, to study the heat transfer characteristics of conduction, convection, radiation for heatsink, we considered the heat transfer characteristics of power electronics(IGBT). To confirm the power loss of power electronics, we analyzed relationship between the profile current, varies according to the time and the average current of the profile. By this analysis, we proposed the heatsink model of optimization for temperature rise. Also, we varified that the temperature rise of heatsink is same in the profile current, varies according to the time as well as in the average current of the profile. Furthermore, we resulted that the time response and heat resistor for heat sink is stable according to the heatsink's volume.
\end{abstract}

Keywords: Optimal design, Power electronics, Power loss, Heat sink, Temperature rise

\section{Introduction}

As a 3 phase inverter prevails, researches on added problems are also being implemented actively. A power semiconductor module is generally consisted with semi conductor chips, wire bonding connecting chips, a ceramic board chips are bonded on and a base plate supporting a board. These multiple materials have a different coefficient of thermal expansion under versatile temperature conditions and this will cause reduction of lifetime of a power semiconductor as thermal stress is induced into it. In addition, heat dissipation characteristic of a power semiconductor is related with lifetime and failure. The subject in this research is to verify loss of a power semiconductor by monitoring temperature of a heat sink in real time for guaranteeing lifetime of a power semiconductor. A power conversion device is consisted with several components, a power semiconductor for switching, a heat sink for lowering junction temperature of a power semiconductor, a fans attached to a heat sink for enhancing cooling capability and compounds to minimize heat resistance of a power semiconductor and a heat sink. If a single component is unstable, lifetime of a power semiconductor may not be guaranteed as it is optimized into system. While it is important to reduce cost by optimizing a power semiconductor into system and lowering rating, it is also important to enhance reliability by guaranteeing lifetime of a power semiconductor required by industry. Modern society requires minimization and low cost of a power conversion device due to fast development of industrial society. The largest part of a power conversion device is a heat sink. A research on a heat sink model for heat loss of a power semiconductor is quite mandatory to provide a minimized inverter by optimizing a heat sink of a 3-phase power inverter.

\section{Analysis of heat transfer of a power semiconductor}

A characteristic of a power semiconductor and that of a heat sink, such as conduction, convection and radiation, that is attached to a power semiconductor are analyzed. Heat flows to transfer from high temperature objects to low temperature ones according to the $2^{\text {nd }}$ law of thermodynamics. There are 3 types of heat transfer, conduction, convection 
and radiation. Heat transfer by conduction is transfer of energy from more active particles to less active ones by interaction of particles in objects. Transfer in a solid is combination of molecular vibration in lattice and energy transfer of free electron. Heat transfer rate through objects differs not only in temperature gap but also in shape, thickness and materials of the object. Fourier's law of heat transfer is represented by formula 1. Heat transfer rate by conduction to certain direction is proportionate to the temperature slope and heat is conducted to the direction temperature is reduced and area $\mathrm{A}$ is always at right angle to the direction of heat transfer.

it $-\mathrm{KA}$ an

where, $\mathrm{K}=$ thermal conductivity of medium in watts/m-K or watts/in

$A=$ cross-sectional area of medium normal to the heat flow path ${ }^{i n^{2}}$ or $\operatorname{cm}^{2}$

$\mathrm{T}=$ temperature of medium in

$\mathrm{X}=$ position along the medium in inch or $\mathrm{cm}$

$\mathrm{t}=$ time in seconds

$\mathrm{q}=$ heat generated per unit volume in joule $/ \mathrm{cm}^{2}$

Thermal characteristics by transfer of a power semiconductor are similar to electrical ones. Temperature change has similarity relation with voltage change. Heat flow rate is similar to current flow. Therefore heat resistance has similarity with electric resistance $\mathrm{R}$. In addition, heat conductivity $\mathrm{K}$ has similarity relation with electric conductivity. A power module is composed of versatile materials and overall heat resistance is addition of each heat resistance like serial connection of electric resistance. In case $\mathrm{N}$ numbers of heat resistances are connected serially, equivalente thermal resistance $\left({ }_{\mathrm{t}}^{\mathrm{t} g \mathrm{~d} t \mathrm{r}}\right)$ is represented as formula 2 .

$0_{\text {tquly }}=0_{1}+0_{2}+0_{3}+\cdots+0_{\mathrm{S}}$

$T_{l 1-1}=T_{\text {heat sink }}+Q_{k} \Sigma_{l i l-1 b s}$

Where, $T_{1:-1}=$ temperature at interface of layer $\mathrm{j}$ and $\mathrm{j}-1$

$\sum \theta_{j-1: b s}=$ sum of thermal resistance from interface of layer $\mathrm{j}$ and $\mathrm{j}-1$ to the heat sink

In this case, temperature on specific location is represented as formula 3. In figure 1, internal thermal resistance of a power module is indicated with electric resistance having similarity. Temperature of two layers connected cannot be discontinuous. Therefore, taking figure 1 for example, temperature of bottom area of a chip(die) is same with that on the top of bonding material. If there are one and more heat paths from heat source to around a power module, equivalence heat resistance is deemed equivalence parallel of each heat resistance. This has similarity with parallel circuit of electric resistance. If there are number $\mathrm{N}$ of heat paths, equivalente thermal resistance $\left({ }^{\theta_{\text {equlv }}}\right)$ is represented as formula 4.

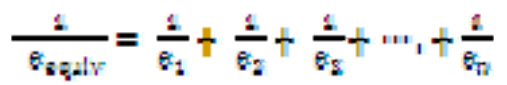

Thermal flow analysis under transient state is represented as RC electric circuit in figure 2 and 3 . Voltage of a capacitor $\left({ }^{V_{C}}\right)$ is charged from $0[\mathrm{~V}]$ to final value $\left({ }^{V_{0}}\right)$ in an exponential function as in formula 5.

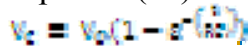

Temperature rise value $(\Delta \mathrm{T})$ across the material can be calculatgd by formula 6 and thermal time constant( ) under steady state can be represented by multiplying thermal resistance( ) with thermal capacity $\left({ }^{C}\right)$ as in formula 7 .

$\Delta T=T_{2}\left(1-a^{-i} \frac{1}{m}\right)$

Where, $\Delta \mathrm{T}=$ temperature difference

$\mathrm{T}_{\boldsymbol{E}}=$ temperature of steady state

$\tau=\theta \mathrm{C}_{\mathrm{f}}$

From a curve of an exponential function in figure 3, time to reach $99.9[\%]$ from formula 8 is obtained.

$\frac{\pi}{7_{\mathrm{E}}}=1-\varepsilon^{-\frac{5}{T}}=0.999$

\section{Analysis of temperature characteristic of a heat sink and modeling}

\subsection{Analysis of temperature characteristic of a heat sink for an inverter}

In figure 4 , the fast time constants of thermal resistance of a power semiconductor( ${ }^{\mathrm{i}}$ ) and slow time constants of thermal resistance of a heat $\operatorname{sink}\left({ }^{\mathrm{T}} \mathrm{h}\right)$ are indicated. The time constant of thermal resistance of a power 
semiconductor $\left(\mathbb{T}_{i}\right)$ is fast with several ten milli-seconds, but the time constant of a heat $\operatorname{sink}\left({ }^{\mathrm{h}}\right)$ is rather slow with several ten minutes.

In order to identify the power loss of a power semiconductor by monitoring temperature on a heat sink in real time, measurement and analysis of heat rise of a heat sink by electric load profiles and analysis of relation between average current and load current profiles are performed and modeling of a heat sink shall be provided un optimizing a heat sink and monitoring temperature of a heat sink. The result of temperature rise test is indicated in figure 5.

The slope is obtained by getting time to saturation after getting functional equation with non-linear curve fitting by dividing temperature rise range in 20 for reliability of data. Equation and shape of curve fitting function of current profile \#1 in table 1 are indicated in figure 6. Figure 7 is the result of temperature rise test and equation and shape of curve fitting function of current profile $\# 3$ are indicated in figure 8.

\subsection{Experimental analysis of temperature rise of profile \#1 and \#3}

Analysis of temperature rise experiment probes that slope value divided in 20 is getting smaller in proportion average current is getting smaller in table 3. Slope (calculation) of profile \#3 is calculated by multiplying slope of profile \#1 with ratio of average current of profile \#1 to that of profile \#3. In this case, it is seen that an error rate falls within $10[\%]$ in range except saturation are.

Average current ratio is useful when calculating temperature of a heat sink. This means that if The average current is known, temperature rise of a heat sink can be calculated with average current value ratio even though different sizes of current profiles are induced into load for a certain period.

In profile \#1 and \#3, functional equation of non-linear curve fitting is obtained from table 3 with formula 9.

$\mathrm{\gamma}=\mathrm{\gamma}_{2}+A_{2}\left(1-\mathrm{a}\left(-\frac{\mathrm{x}}{\mathrm{s}}\right)+A_{2}\left(1-\mathrm{a} \cdot-\frac{\mathrm{x}}{\mathrm{s}}\right)\right.$

Where, $\mathrm{Y}=$ temperature of heat sink, $\mathrm{x}=$ time[second], ${ }_{0} \mathrm{Y}_{0}=$ initial temperature of heat sink

Thermal resistance at a measuring point of temperature rise of a heat sink can be identified designed with parallel circuit. Each item can be replaced with R-C circuit and thermal time constant can be identified from functional equation calculated from non-linear curve fitting function. Accurate thermal time constant is difficult to find when doing temperature rise test. Because it takes quite a long time to reach 5 tau of exponential function. In this case, time to reach saturation temperature can be easily obtained by functional equation of non-linear curve fitting. In equation of profile $\# 1$ and \#3, average current ratio can be seen from ratio of A1 to A2 that represent size. Time corresponding to time constant of each profile \#1 and \#3 is represented as in figure 11 and table 5. In table 5, although current profile \#1 and \#3 have different current value, it is identified time to reach single, double and triple of time constant is almost identical.

Once modeling size and measuring location of temperature on a heat sink are fixed when modeling a heat sink, the principle that time constant is the same even in different current profile can be used in temperature simulation and optimization of a heat sink.

That means as time constant is always consistent and only saturation temperature is different, period of average current can be time constant of a heat sink even though volume of induced power is different.

\section{Conclusion}

In order to identify temperature characteristic of a heat sink, different ratings of current profiles are induced into a 3-phase AC reactor and temperature rise of a heat sink where a power semiconductor (IGBT) is bonded is monitored. At this time, time and saturation temperature are analyzed by each current profile until temperature of a heat sink is saturated. By doing this, it is identified that once average current is known, temperature rise of a heat sink can be calculated with average current value ratio even though different sizes of electric profiles are induced in load. And period of average current can be time constant of a heat sink as time constant is always consistent and only size of saturation temperature is different even though volume of induced power is different. Once modeling size and measuring location of temperature on a heat sink are fixed when modeling a heat sink, the principle that time constant is the same even in different current profile can be used in temperature simulation and optimization of a heat sink.

\section{Acknowledgement}

The present research has been conducted by the Research Grant of Seoil University in 2007

\section{References}

C.K.Loh, D.Nelson, D.JChou. (2001). Thermal characterization of fan-heat sink systems in miniature axial fan and micro blower airflow, Semiconductor Thermal Measurement and Management, 2001. Seventeenth Annual IEEE Symposium 20-22 March 2001 Page(s):111 - 116. 
F.Auerbach, A.Lenniger. (1997). Power-Cycling-Stability of IGBT-Modules, IAS '97. Industry Applications Conference, IEEE Conference Record. Vol.2, pp.1248-1252, Oct. 1997.

H.De Lambilly, H.Keser. (1992). Failure Analysis of Power modules: A Look at the Packaging and Reliability of large IGBT's, Thirteenth IEEE/CHMT International. Electronics Manufacturing Technology Symposium, 1992. September 28-30, 1992, pp.366-370.

J.Richard Culham, Yuri S. Muzychka. (2001). Optimization of Plate Fin Heat Sinks Using Entropy Generation Minimization, Components and Packaging Technologies, IEEE Transactions on [see also Components, Packaging and Manufacturing Technology, Part A: Packaging Technologies, IEEE Transactions on] Vol 24, Issue 2, June 2001 Page(s):159 - 165

M.Ciappa, F.Carbognani, W.Fichtner. (2003). Lifetime modeling of Thermo-mechanics-Related Failure Mechanisms in High Power IGBT Modules for Traction Applications, Proc. ISPSD '03,Power Semiconductor Devices and ICs, 15th April 2003, pp.295-298.

M.ini, R.L.Webb. (2003). Heat rejection limits of air cooled plane fin heat sinks for computer cooling, Components and Packaging Technologies, IEEE Transactions on [see also Components, Packaging and Manufacturing Technology, Part A: Packaging Technologies, IEEE Transactions on] Vol 26, Issue 1, March 2003 Page(s):71 - 79

M.Iyengar, A.Bar-Cohen. (2003). Least-energy optimization of forced convection plate-fin heat sinks, Components and Packaging Technologies, IEEE Transactions on [see also Components, Packaging and Manufacturing Technology, Part A: Packaging Technologies, IEEE Transactions on] Vol 26, Issue 1, March 2003 Page(s):62-70

Mauro Ciappa and Wolfgang Fichtner. (2000). Lifetime Prediction of IGBT Modules for Traction Applications, Proc. IEEE International Reliability Physics Symposium, San Jose, 2000, pp. 210-216.

V.A.Sankaran, C.Chen, C.S.Avant, X.Xu. (1997). Power Cycling Reliability of IGBT Power modules, IAS '97., Industry Applications Conference. Vol.2, Oct. 1997, pp.1222-1227.

William W. Sheng and Ronald P. Colino. Power Electronics Modules Design and Manufacture, CRC PRESS. 
Table 1. Current value and time period for each load current profile

\begin{tabular}{|c|c|c|c|c|c|c|c|}
\hline & \multicolumn{7}{|c|}{ Load current profile \#1 } \\
\hline & \multicolumn{4}{|c|}{ Full load up direction } & \multicolumn{3}{|c|}{ Full load down direction } \\
\hline & $\mathrm{T} 1$ & $\mathrm{~T} 2$ & $\mathrm{~T} 3$ & T4 & T5 & T6 & $\mathrm{T} 7$ \\
\hline Current $[\mathrm{A}]$ & 27 & 27 & 15 & 7.5 & 7.5 & 9 & 22.5 \\
\hline \multirow[t]{4}{*}{ Time $[$ second $]$} & 1 & 0.25 & 15.5 & 3.25 & 1.25 & 15.5 & 3.25 \\
\hline & \multicolumn{7}{|c|}{ Load current profile \#1-1( Average current ) } \\
\hline & \multicolumn{4}{|c|}{ Full load up direction } & \multicolumn{3}{|c|}{ Full load down direction } \\
\hline & $\mathrm{T} 1$ & $\mathrm{~T} 2$ & $\mathrm{~T} 3$ & $\mathrm{~T} 4$ & $\mathrm{~T} 5$ & T6 & $\mathrm{T} 7$ \\
\hline Current[A] & & & & 12.48 & & & \\
\hline \multirow[t]{4}{*}{ Time[second] } & 1 & 0.25 & 15.5 & 3.25 & 1.25 & 15.5 & 3.25 \\
\hline & \multicolumn{7}{|c|}{ Load current profile \#3 } \\
\hline & \multicolumn{4}{|c|}{ Full load up direction } & \multicolumn{3}{|c|}{ Full load down direction } \\
\hline & $\mathrm{T} 1$ & $\mathrm{~T} 2$ & $\mathrm{~T} 3$ & T4 & $\mathrm{T} 5$ & T6 & $\mathrm{T} 7$ \\
\hline Current $[\mathrm{A}]$ & 20.25 & 20.25 & 11.25 & 5.63 & 5.63 & 6.75 & 16.88 \\
\hline \multirow[t]{4}{*}{ Time[second $]$} & 1 & 0.25 & 15.5 & 3.25 & 1.25 & 15.5 & 3.25 \\
\hline & \multicolumn{7}{|c|}{ Load current profile \#3-1( Average current ) } \\
\hline & \multicolumn{4}{|c|}{ Full load up direction } & \multicolumn{3}{|c|}{ Full load down direction } \\
\hline & $\mathrm{T} 1$ & $\mathrm{~T} 2$ & $\mathrm{~T} 3$ & $\mathrm{~T} 4$ & $\mathrm{~T} 5$ & T6 & $\mathrm{T} 7$ \\
\hline Current $[\mathrm{A}]$ & \multicolumn{7}{|c|}{9.36} \\
\hline Time[second] & 1 & 0.25 & 15.5 & 3.25 & 1.25 & 15.5 & 3.25 \\
\hline
\end{tabular}


Table 2. Temperature rise and slope values at load current profile \#3

\begin{tabular}{|c|c|c|c|c|}
\hline \multicolumn{5}{|c|}{ Load current profile \#3 } \\
\hline Time[second] & Ideal value [ $\left.{ }^{\circ} \mathrm{C}\right]$ & Measured value $\left[{ }^{\circ} \mathrm{C}\right]$ & Slope(ideal) & Slope(measured) \\
\hline 0.0000 & 27.5000 & 27.5000 & & \\
\hline 33.7500 & 29.2896 & 29.2765 & 0.0530 & 0.0526 \\
\hline 78.7500 & 30.9859 & 31.0530 & 0.0377 & 0.0395 \\
\hline 153.7500 & 32.9166 & 32.8295 & 0.0257 & 0.0237 \\
\hline 243.7500 & 34.6106 & 34.6060 & 0.0188 & 0.0197 \\
\hline 352.5000 & 36.3299 & 36.3825 & 0.0158 & 0.0163 \\
\hline 483.7500 & 38.2015 & 38.1590 & 0.0143 & 0.0135 \\
\hline 615.0000 & 39.9303 & 39.9355 & 0.0132 & 0.0135 \\
\hline 750.0000 & 41.5830 & 41.7120 & 0.0122 & 0.0132 \\
\hline 918.7500 & 43.4860 & 43.4885 & 0.0113 & 0.0105 \\
\hline 1102.5000 & 45.3704 & 45.2650 & 0.0103 & 0.0097 \\
\hline 1290.0000 & 47.1101 & 47.0415 & 0.0093 & 0.0095 \\
\hline 1496.2500 & 48.8312 & 48.8180 & 0.0083 & 0.0086 \\
\hline 1736.2500 & 50.6072 & 50.5945 & 0.0074 & 0.0074 \\
\hline 2002.5000 & 52.3263 & 52.3710 & 0.0065 & 0.0067 \\
\hline 2295.0000 & 53.9510 & 54.1475 & 0.0056 & 0.0061 \\
\hline 2748.7500 & 56.0151 & 55.9240 & 0.0045 & 0.0039 \\
\hline 3270.0000 & 57.8394 & 57.7005 & 0.0035 & 0.0034 \\
\hline 3870.0000 & 59.3932 & 59.4770 & 0.0026 & 0.0030 \\
\hline 5080.3700 & 61.3427 & 61.2535 & 0.0016 & 0.0015 \\
\hline 15118.0000 & 63.4505 & 63.0300 & 0.0002 & 0.0002 \\
\hline
\end{tabular}


Table 3. Comparison of the slope between measured value and calculated value at current profile \#3

\begin{tabular}{|c|c|c|c|}
\hline Profile \#1 & \multicolumn{2}{|c|}{ Profile \#3 } & \multirow{2}{*}{$\begin{array}{c}\text { Error ratio(measured vs calculated) } \\
\text { slope }\end{array}$} \\
\hline Slope(measured) & Slope(measured) & Slope(calculated) & \\
\hline 0.0708 & 0.053 & 0.0531 & 1.00189 \\
\hline 0.0473 & 0.0377 & 0.035475 & 0.94098 \\
\hline 0.0307 & 0.0257 & 0.023025 & 0.89591 \\
\hline 0.0233 & 0.0188 & 0.017475 & 0.92952 \\
\hline 0.0208 & 0.0158 & 0.0156 & 0.98734 \\
\hline 0.0193 & 0.0143 & 0.014475 & 1.01224 \\
\hline 0.0179 & 0.0132 & 0.013425 & 1.01705 \\
\hline 0.0165 & 0.0122 & 0.012375 & 1.01434 \\
\hline 0.015 & 0.0113 & 0.01125 & 0.99558 \\
\hline 0.0137 & 0.0103 & 0.010275 & 0.99757 \\
\hline 0.0124 & 0.0093 & 0.0093 & 1.00000 \\
\hline 0.0112 & 0.0083 & 0.0084 & 1.01205 \\
\hline 0.0099 & 0.0074 & 0.007425 & 1.00338 \\
\hline 0.0086 & 0.0065 & 0.00645 & 0.99231 \\
\hline 0.0072 & 0.0056 & 0.0054 & 0.96429 \\
\hline 0.0058 & 0.0045 & 0.00435 & 0.96667 \\
\hline 0.0046 & 0.0035 & 0.00345 & 0.98571 \\
\hline 0.0033 & 0.0026 & 0.002475 & 0.95192 \\
\hline 0.0019 & 0.0016 & 0.001425 & 0.89063 \\
\hline 0.0002 & 0.0002 & 0.00015 & 0.75000 \\
\hline
\end{tabular}


Table 4. Comparison of constant value of functional equation between profile \#1 and \#3

\begin{tabular}{|c|c|c|}
\hline & $\# 1$ & $\# \mathbf{3}$ \\
\hline $\mathrm{Y}_{\curvearrowleft}$ & 23.74 & 27.50 \\
\hline $\mathrm{A} 1$ & $\mathbf{4 5 . 8 6}$ & $\mathbf{3 . 1 7}$ \\
\hline$\tau_{1}$ & 1870.15 & $\mathbf{7 1 . 4 9}$ \\
\hline $\mathrm{A} 2$ & $\mathbf{3 . 5 0}$ & 32.78 \\
\hline$\tau_{2}$ & 53.71 & 1854.30 \\
\hline
\end{tabular}

Table 5. A comparison of the time constant by using functional equation between profile $\# 1$ and \#3

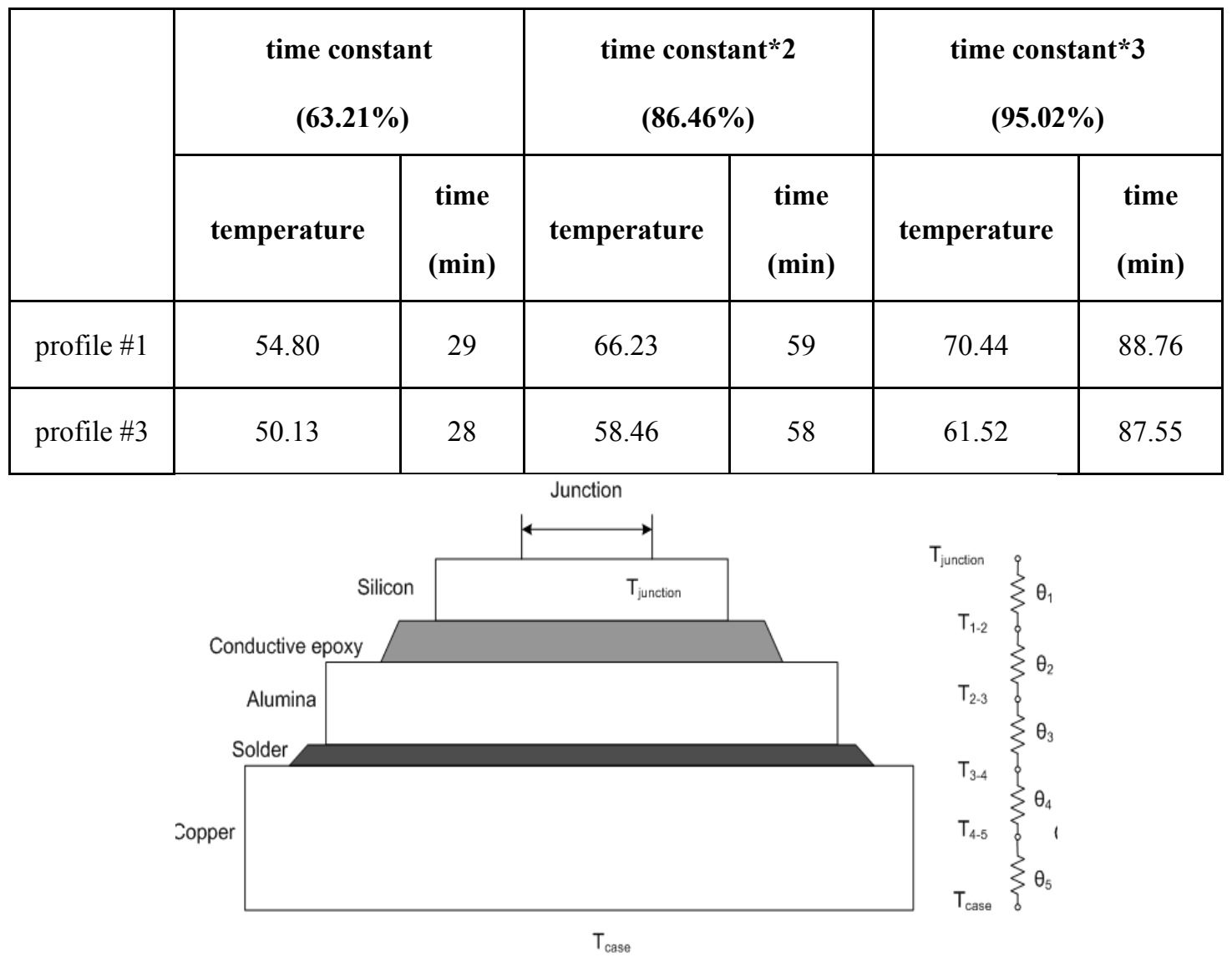

Figure 1. Stackup of power module with electrical analog

where, $\theta_{1}=$ thermal resistance of die

$\theta_{2}=$ thermal resistance of die attach

$\theta_{3}=$ thermal resistance of substrate

$\theta_{4}=$ thermal resistance of substrate attach

$\theta_{5}=$ thermal resistance of package

$\mathrm{T}_{\mathrm{j}}=$ junction temperature

$\mathrm{T}_{\text {case }}=$ case temperature 


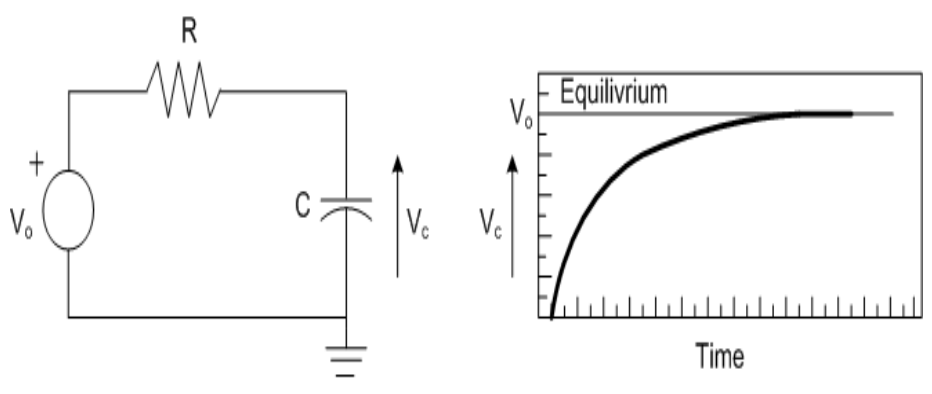

Figure 2. Resistor-capacitor network and time response

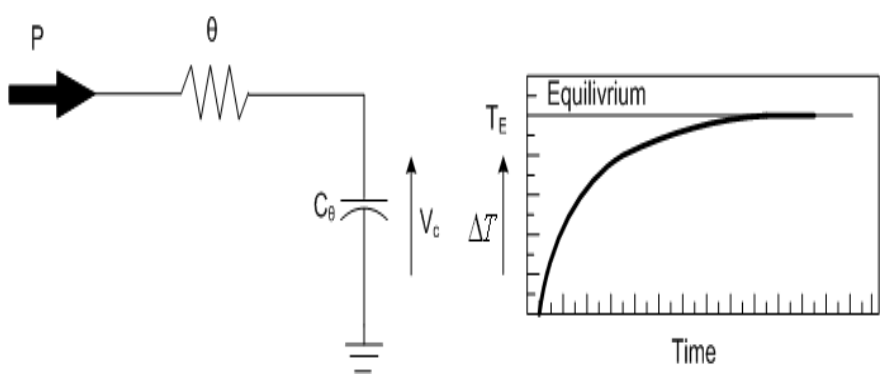

Figure 3. Thermal network and time response

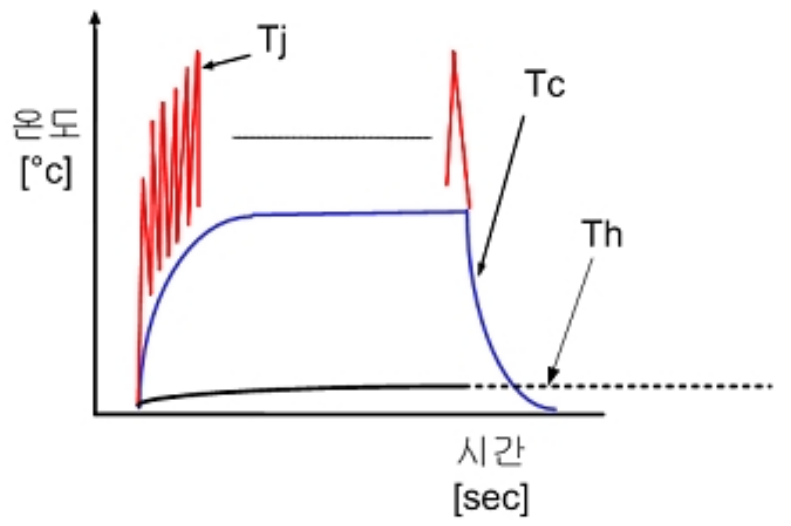

Figure 4. A comparison of time response of thermal resistance of power electronics and Heat-sink 


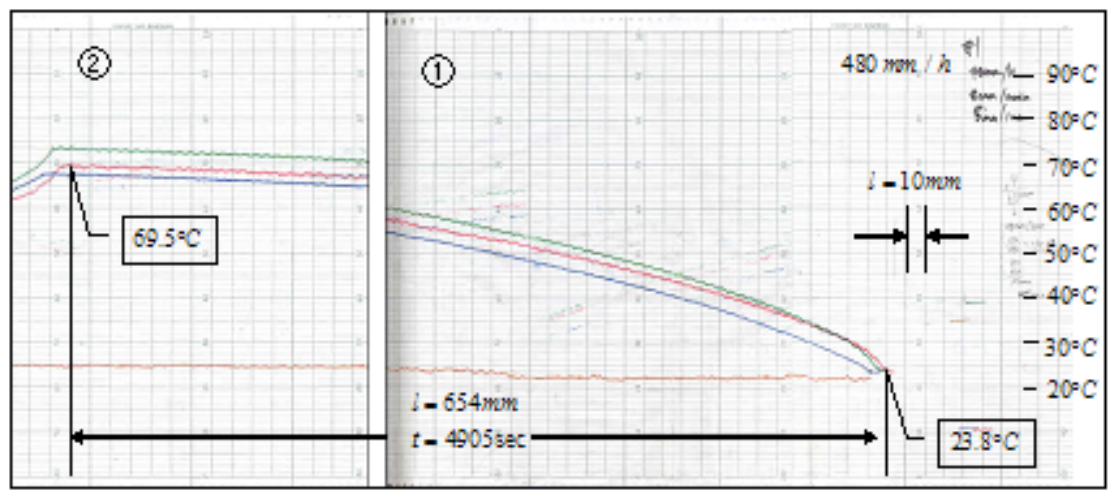

Figure 5. The test of temperature rise at current profile \#1

$\left[{ }^{\circ} \mathrm{C}\right]$

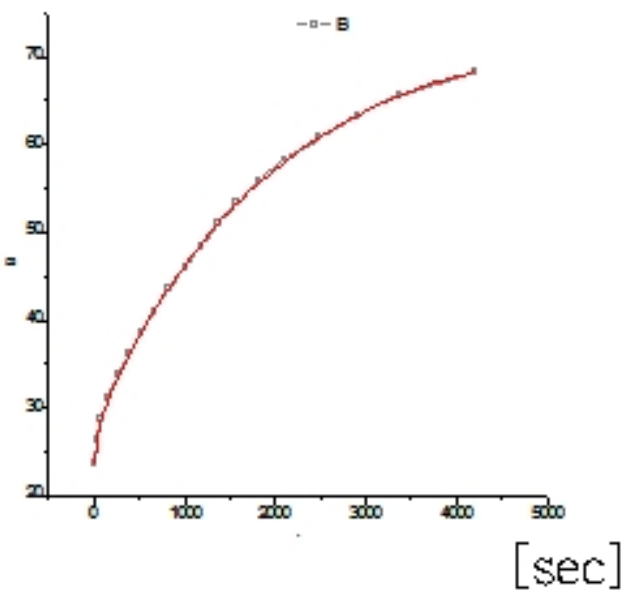

Equation: $y=y+A 1+(1-\exp (-x t 1))$

$+A 2 *(1-\exp (-x+2))$

$\mathrm{Cri}^{\wedge} 2 \mathrm{DOF}=0,03297$

$\mathrm{R} 2=0,99987$

yo 23,73941

A1 $\quad 45,86497$

t1 1870,14838

A2 $\quad 346589$

t2 $\quad 537126$

Figure 6. Functional equation and graph by using non-linear curve fitting for load current profile \#1

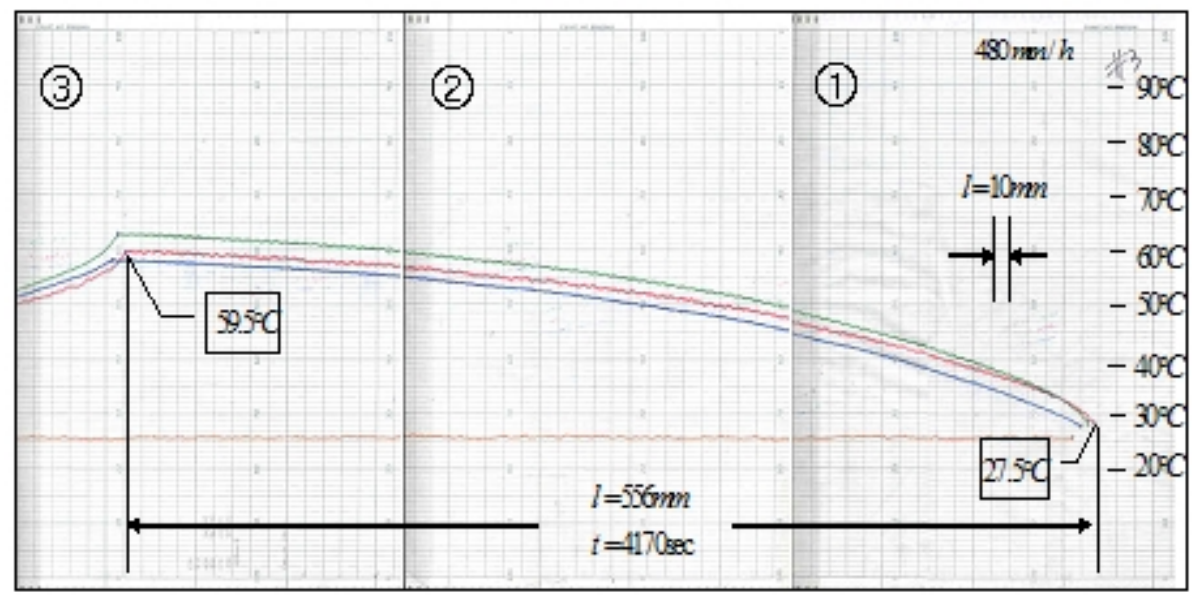

Figure 7. The test of temperature rise at load current profile \#3 


\section{$\left[{ }^{\circ} \mathrm{C}\right]$}

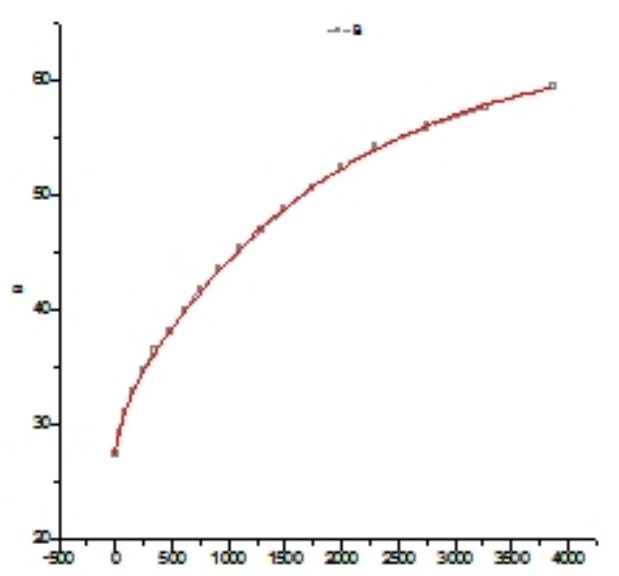

Eqdiory $=y 0+A 1+(1-e d(-x t 1))$

$+A 2(1-e x(-x+2))$

Gizor $=0,0002$

$\mathrm{R}^{\prime} 2=0.9093$

yo 27,5042

A1 $\quad 3.17318$

t1 $\quad 71.49066$

A2 $3278 \times 59$

†2 1842991

\section{[sec]}

Figure 8. Functional equation and graph by using non-linear curve fitting for load current profile \#3

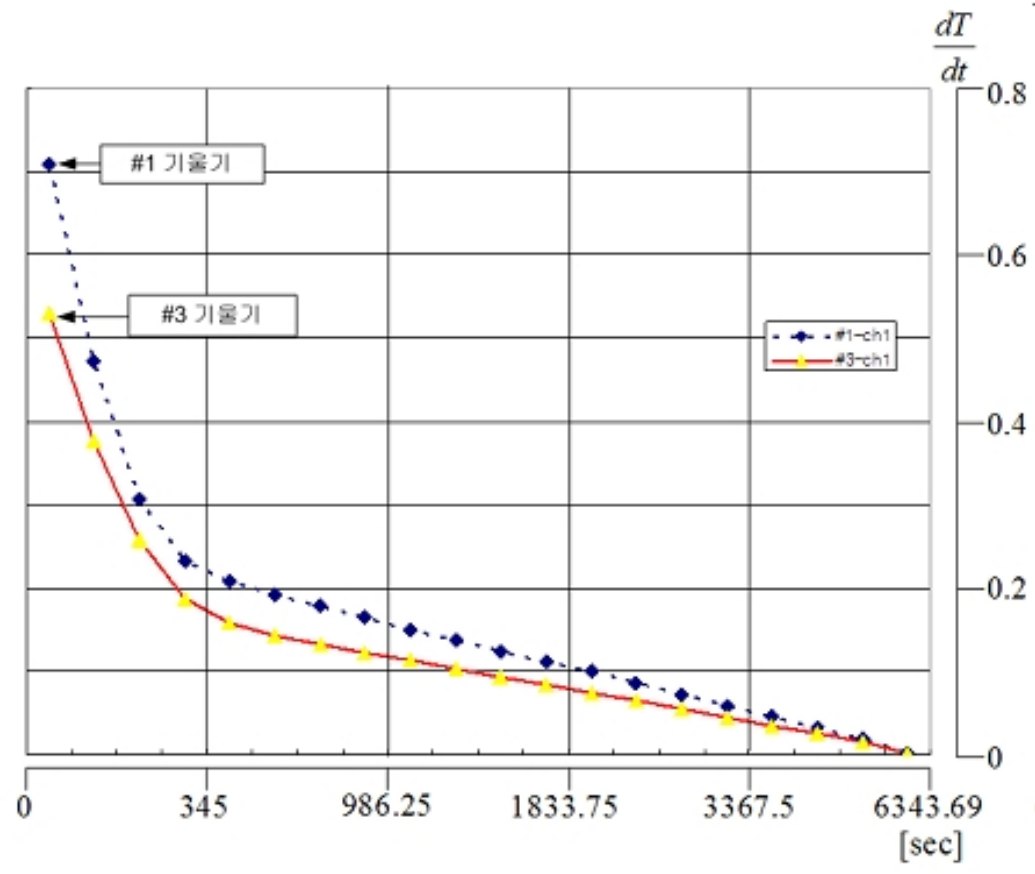

Figure 9. Comparison of slope of the temperature rise between profile \#1 and \#3 


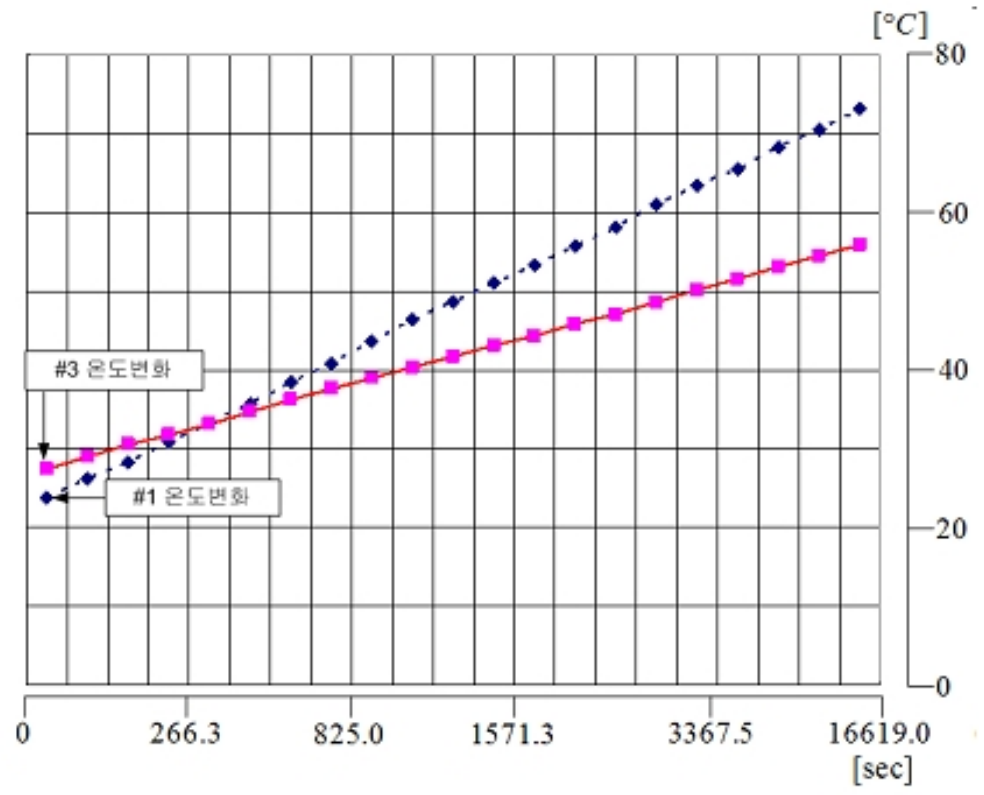

Figure 10. Comparison of the temperature rise between profile \#1 and \#3

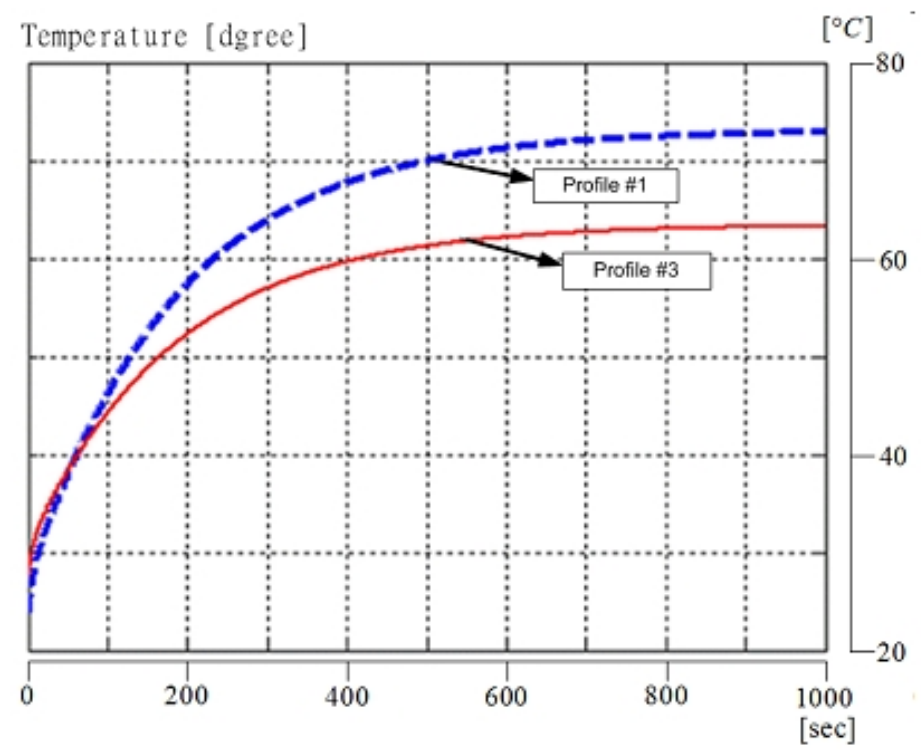

Figure 11. A Comparison of the temperature rise by using functional equation between profile \#1 and \#3 